\title{
NAD-Coupled Enzymatic Oxidation of O-Ethyl O-p-Nitrophenyl Phenylphosphonothioate (EPN) to Its Oxygen Analog with Liver Microsomes of Rats
}

\author{
Seiyu SUGIYAMA, Takashi IGARASHI, Koichi UENO, \\ Tetsuo SATOH* and Haruo KITAGAWA \\ Laboratory of Biochemical Pharmacology and Biotoxicology. \\ Department of Drug Fvaluation and Toxicological Sciences, \\ Faculty of Pharmaceutical Sciences, Chiba University. \\ 1-33 Yayoi-cho. Chiba 260. Japan \\ Accepted November 24, 1984
}

\begin{abstract}
O-Ethyl O-p-nitrophenyl phenylphosphonothioate (EPN)-induced inhibition of rat liver microsomal carboxylesterase (CEase) and formation of $\mathrm{O}$ ethyl O-p-nitrophenyl phenylphosphonate (EPNoxon), an oxygen analog of EPN. were enhanced remarkably by addition of NAD in vitro. This potentiation of the anti-CEase action of EPN by NAD was significantly inhibited by addition of SKF 525-A or potassium thiocyanate ( $K S C N$ ); and a simultaneous decrease in cytochrome P-450 contents was also observed. Addition of N-ethylmaleimide (NEM) at various concentrations inhibited potentiation of the anti-CEase action of EPN by NAD in parallel with inhibition of liver microsomal dehydrogenase activities. In conclusion. NAD was enzymatically reduced to NADH, a cofactor of microsomal dehydrogenase(s), and then formation of EPNoxon through microsomal cytochrome P-450-coupled monooxygenase was accelerated. Consequently, inhibition of CEase by EPN was potentiated.
\end{abstract}

Our previous papers $(1,2)$ and unpublished observation (S. Sugiyama et al.) showed that the anti-CEase action of organophosphorothioate insecticides such as EPN and parathion was potentiated by addition of NAD in the presence of liver microsomes without an added NADPH-generating system. This phenomenon has been referred to as the "NAD-effect" in a series of these studies. The NAD-effect was not seen when organophosphate insecticides such as paraoxon was added to the incubation mixture. In addition, no NAD-effect was observed when pure esterase (E.C. 3.1.1.1) instead of liver microsomes was used as the enzyme source. Addition of sulfhydryl-blocking reagents such as NEM and PCMB diminished the NAD-effect (2). Further, there is a close

\footnotetext{
* Present address: Department of Pharmacology. Tokyo College of Pharmacy, 1432-1 Horinouchi, Hachioji. Tokyo 192-03, Japan
}

relation between the chemical structure of organophosphorothioate insecticides and occurrence of the NAD-effect (S. Sugiyama et al., unpublished observation). From these findings, it is speculated that the NAD-effect may be induced by organophosphate formation through microsomal enzymes including dehydrogenase(s) and the cytochrome P-450-coupled reaction. The present study was, therefore, designed to investigate the further detailed mechanism of the NADeffect in which microsomal dehydrogenase(s) may be involved.

\section{Materials and Methods}

Animal: Adult male rats of the Wistar strain, weighing $200-230 \mathrm{~g}$, were used. They were maintained on standard laboratory chow (Oriental Yeast Co., MF) and tap water ad libitum under a condition of constant room temperature $\left(23^{\circ}-25^{\circ} \mathrm{C}\right)$ for at least one week before use. 
Preparation of liver microsomes: The rats were sacrificed by decapitation, and the livers were immediately removed. Perfusion with $1.15 \% \mathrm{KCl}$ was carried out to remove the blood immediately. Livers were homogenized in a Potter-Elvehjem homogenizer with a motor-driven Teflon pestle to give a $33 \%(\mathrm{w} / \mathrm{v})-1.15 \% \mathrm{KCl}$ homogenate. The homogenate was centrifuged at $9,000 \times \mathrm{g}$ for $20 \mathrm{~min}$. The microsomal pellet was obtained by recentrifugation at $105,000 \times \mathrm{g}$ for $60 \mathrm{~min}$. The pellet was suspended in $1.15 \% \mathrm{KCl}$ before use.

The liver microsomes were solubilized using sodium cholate according to the method of Imai and Sato (3) in the presence of glycerol, EDTA and dithiothreitol.

Biochemical analyses: CEase activity was determined using the colorimetric assay procedure based on conversion of isocarboxazid to benzylhydrazine $(\mathrm{BZH})$ as described previously $(1,4)$. Cytochrome P-450 contents in liver microsomes were measured by the method of Omura and Sato (5). Aldehyde dehydrogenase activity was determined by the method of Nakayasu et al. (6) using decylaldehyde as a substrate. Protein concentration of liver microsomes was determined by the method of Lowry et al. (7).

Determination of EPNoxon: EPNoxon formed in the incubation mixture containing liver microsomes, EPN, $\operatorname{NAD}(P)(H)$ and other reagents without added NADPHgenerating system after 30 min-incubation was determined by gas chromatography-mass spectrometry as reported earlier (1).

\section{Results}

Changes in NAD-effect on liver microsomes by solubilization: NAD-mediated potentiation of the anti-CEase action of EPN was determined using microsomes solubilized by sodium cholate $(0.05 \mathrm{mg}-1$ $\mathrm{mg} / \mathrm{mg}$ microsomal protein).

As shown in Table 1, the NAD-effect was not seen when $0.2 \mathrm{mg}$ of sodium cholate was added to the pellet fraction (ppt) and seen when $1 \mathrm{mg}$ of sodium cholate was added to the supernatant (sup). This study revealed that an unknown factor(s) in liver microsomes that is required for the manifestation of the NAD-effect seemed to be a protein which could be solubilized by sodium cholate.

Determination of EPNoxon formed by addition of NAD in the supernatant after solubilization of microsomes: In order to clarify the NAD-effect, NAD-mediated formation of EPNoxon from EPN was studied when the supernatant of the microsomes solubilized by sodium cholate was used as the enzyme source. In the inhibition studies, NEM was used as a sulfhydryl-blocking reagent (2). Phenylmethylsulfonyl fluoride (PMSF) (8) and EDTA $(8,9)$ were used to inhibit binding of EPNoxon to both CEase and A type-esterase (10) which was responsible for hydrolysis of EPNoxon.

As shown in Table 2, NAD-mediated formation of EPNoxon from EPN was clearly increased, compared to that in the absence of NAD, while addition of NEM to the incubation mixture showed no EPNoxon

Table 1. Effects of treatment of liver microsomes with cholate on the potentiation of EPN-induced inhibition of carboxylesterase by NAD

\begin{tabular}{|c|c|c|c|c|c|c|}
\hline \multirow{3}{*}{$\begin{array}{l}\text { Cholate } \\
\text { added } \\
\text { (mg/mg } \\
\text { protein) }\end{array}$} & \multicolumn{6}{|c|}{$\begin{array}{c}\text { Carboxylesterase activity } \\
\text { (nmoles BZH formed } / \mathrm{mg} \text { protein } / 30 \mathrm{~min} \text { ) }\end{array}$} \\
\hline & & ppt. & & & sup. & \\
\hline & Control & EPN & $E P N+N A D$ & Control & EPN & $E P N+N A D$ \\
\hline None & $52.2 \pm 3.3$ & $48.6 \pm 2.5$ & $12.0 \pm 1.4^{*}$ & $27.9 \pm 9.8$ & $18.5 \pm 7.1$ & $13.2 \pm 2.5$ \\
\hline 0.05 & $50.5 \pm 2.6$ & $46.8 \pm 2.3$ & $11.6 \pm 1.7^{*}$ & $53.6 \pm 1.6$ & $33.5 \pm 6.3$ & $33.3 \pm 6.9$ \\
\hline 0.2 & $40.1 \pm 8.8$ & $37.4 \pm 8.1$ & $28.1 \pm 5.8$ & $103.3 \pm 4.7$ & $86.1 \pm 3.5$ & $82.1 \pm 4.2$ \\
\hline 1.0 & $9.9 \pm 3.5$ & $7.9 \pm 3.0$ & $7.0 \pm 2.3$ & $135.9 \pm 11.5$ & $132.1 \pm 10.0$ & $75.6 \pm 15.0^{*}$ \\
\hline
\end{tabular}

The concentrations of EPN and NAD used were $1 \times 10^{-5} \mathrm{M}$ and $5 \times 10^{-5} \mathrm{M}$, respectively. Each value is the mean $\pm S$. E. from five experiments. ${ }^{*} P<0.01$, statistically significant difference from each EPN group. 
Table 2. Effects of NAD on the enzymatic formation of EPNoxon from EPN in the supernatant of liver microsomes after cholate treatment

\begin{tabular}{lcc}
\hline Addition & EPNoxon formed $\left(10^{-7} \mathrm{M}\right)$ & \%(EPNoxon/EPN added) \\
\hline EPN & $N . \mathrm{D}$. & - \\
EPN + NAD & $1.28 \pm 0.15$ & 0.85 \\
EPN + NAD + NEM & N.D. & - \\
EPN + NAD + PMSF+EDTA & $1.48 \pm 0.12$ & 0.99 \\
EPN + NAD + PMSF+EDTA+NEM & N.D. & - \\
\hline
\end{tabular}

N.D.: less than $0.5 \times 10^{-7} \mathrm{M}$. The initial concentration of EPN added was $1.5 \times 10^{-5} \mathrm{M}$. The concentrations of NAD, NEM, PMSF and EDTA used were $7.5 \times 10^{-5} \mathrm{M}, 1.5 \mathrm{mM}, 1 \mathrm{mM}$ and $4.5 \times 10^{-5} \mathrm{M}$ in $5 \mathrm{ml}$ of incubation mixture, respectively. Liver microsomes were solubilized by addition of sodium cholate (1 $\mathrm{mg} / \mathrm{mg}$ protein) at $0^{\circ} \mathrm{C}$ for $30 \mathrm{~min}$. Each value is the mean $\pm S$. E. from four experiments.

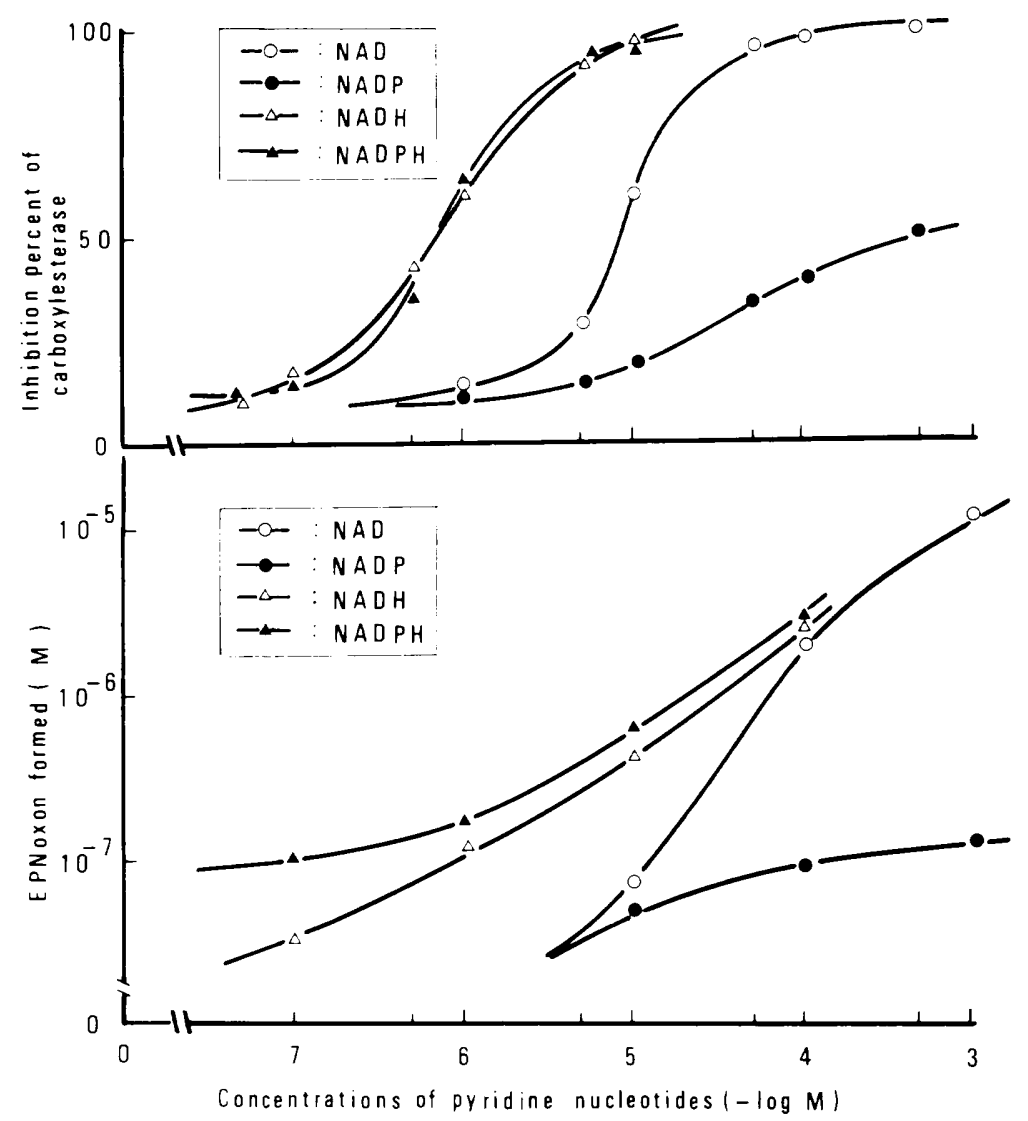

Fig. 1. Effects of pyridine nucleotides on EPN-induced inhibition of liver microsomal carboxylesterase and formation of EPNoxon in vitro. The initial concentration of EPN added was $1 \times 10^{-5} \mathrm{M}$ in $1.5 \mathrm{ml}$ of incubation mixture for the carboxylesterase assay and $1.5 \times 10^{-5} \mathrm{M}$ in $1 \mathrm{ml}$ of incubation mixture for the EPNoxon assay. Each point is the mean of three experiments.

formation. Conversely, EPNoxon formation was slightly increased by addition of both PMSF and EDTA.

Effects of pyridine nucleotides on EPN- induced inhibition of CEase and formation of EPNoxon: The correlation of potentiation of the anti-CEase action of EPN and the formation of EPNoxon from EPN was 
studied when $N A D(H)$ or $\operatorname{NADP}(H)$ was added.

As shown in Fig. 1, inhibition of liver microsomal CEase by EPN was significantly potentiated by addition of NAD $\left(5 \times 10^{-6}\right.$ $\left.5 \times 10^{-5} \mathrm{M}\right), \quad \mathrm{NADH}\left(5 \times 10^{-7}-5 \times 10^{-6} \mathrm{M}\right)$ and NADPH $\left(5 \times 10^{-7}-5 \times 10^{-6} \mathrm{M}\right)$, and it was only slightly potentiated by NADP $\left(5 \times 10^{-5}-5 \times 10^{-4} \mathrm{M}\right)$. On the other hand, the increase of EPNoxon formation following addition of the four pyridine nucleotides was closely related to the degree of pyridine nucleotides-mediated potentiation of the antiCEase action of EPN. Among these four pyridine nucleotides, NADPH- and NADHinduced potentiation of the anti-CEase actin of EPN could be explained by a direct effect on cytochrome P-450-coupled oxidative desulfuration of EPN to EPNoxon. The potentiation induced by NAD seemed to be due to the formation of EPNoxon through cytochrome P-450-coupled monooxygenase after conversion of NAD to NADH through NAD-dependent microsomal dehydrogenase(s).

Effects of NEM on NAD $(\mathrm{H})$-mediated potentiation of anti-CEase action of EPN and microsomal aldehyde dehydrogenase activity: From the results described above, it

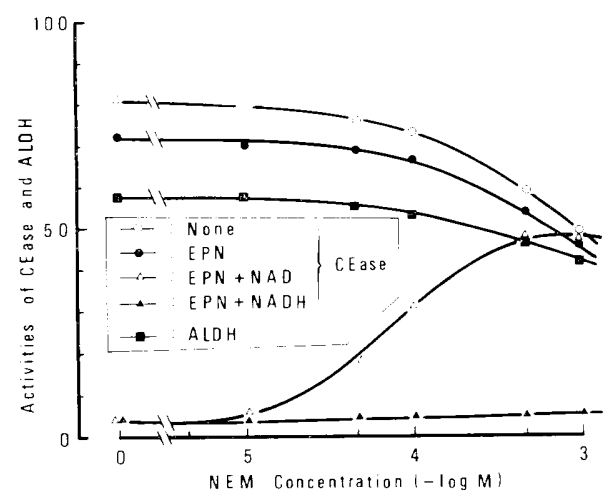

Fig. 2. Effects of $\mathrm{N}$-ethylmaleimide on the NADeffect and microsomal aldehyde dehydrogenase activity. Activities of carboxylesterase (CEase) and aldehyde dehydrogenase (ALDH) are shown as nmoles of BZH formed $/ \mathrm{mg}$ protein $/ 30 \mathrm{~min}$ and nmoles of $\mathrm{NADH}$ formed/mg protein $/ \mathrm{min}$, respectively. The concentrations of EPN and $\operatorname{NAD}(H)$ used were $1 \times 10^{-5} \mathrm{M}$ and $5 \times 10^{-5} \mathrm{M}$, respectively. Each point is the mean of three experiments. is likely that EPNoxon formation from EPN was catalyzed through microsomal cytochrome P-450-coupled monooxygenase. So. involvement of NAD-dependent dehydrogenase(s) in liver microsomes in the NAD-effect was studied. We have already reported that addition of NEM to the incubation mixture containing liver microsomes, EPN and NAD caused a remarkable decrease of the NAD-effect (2). In this study, degree of formation of EPNoxon from EPN in the incubation mixture was decreased (Table 2).

As shown in Fig. 2, the NAD-effect was inhibited by addition of NEM as a function of concentration. In parallel, microsomal aldehyde dehydrogenase activity was also inhibited by addition of NEM: and this was relatively correlated to the inhibition of the NAD-effect. From these findings, it was possible to speculate that inhibition of the production of NADH from NAD through microsomal dehydrogenase(s) caused a decrease of EPNoxon formation.

Effect of disulfiram on NAD $(H)$-mediated potentiation of anti-CEase action of EPN: As shown in Fig. 3. NAD- and NADHmediated potentiation of the anti-CEase action of EPN was inhibited by addition of disulfiram at a concentration of $5 \times 10^{-5} \mathrm{M}$ and $1 \times 10^{-4} \mathrm{M}$, respectively. As disulfiram is

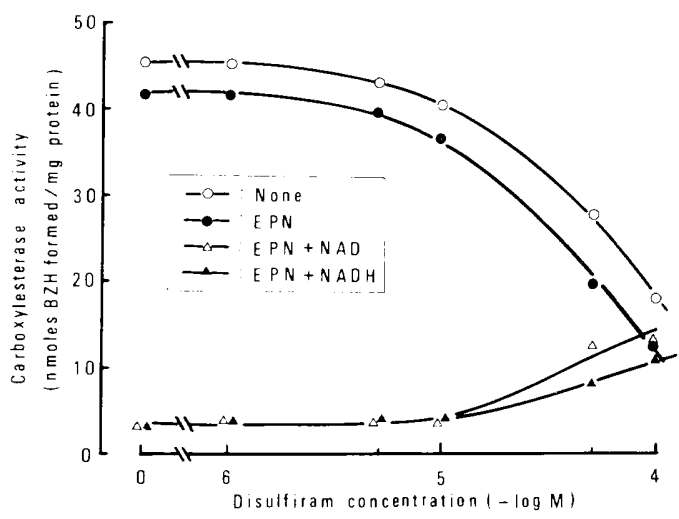

Fig. 3. Effects of disulfiram on $\operatorname{NAD}(\mathrm{H})$-mediated potentiation of the anti-carboxylesterase action of EPN. The concentrations of EPN, NAD and NADH used were $1 \times 10^{-5} \mathrm{M}, 5 \times 10^{-5} \mathrm{M}$ and $5 \times 10^{-5} \mathrm{M}$. respectively. Each point is the mean of three experiments. 
Table 3. Effects of SKF 525-A on NAD-mediated potentiation of the anti-carboxylesterase action of EPN

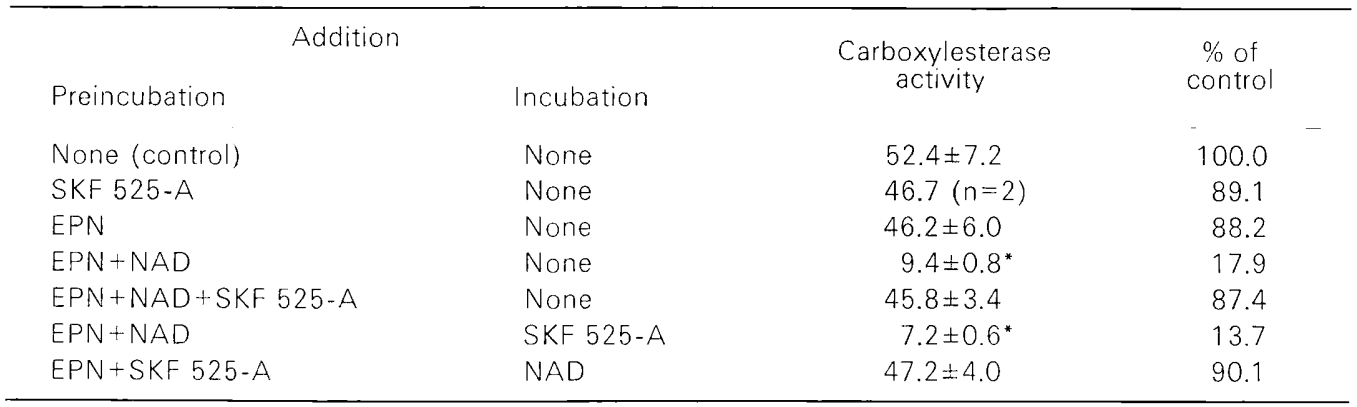

Liver microsomes were preincubated at $37^{\circ} \mathrm{C}$ for $10 \mathrm{~min}$ with $0.5 \mathrm{ml}$ of Tris- $\mathrm{HCl}$ buffer $(\mathrm{pH} \mathrm{8.0)}$ plus the addition listed in the table in a totai volume of $1.0 \mathrm{ml}$. At the end of preincubation, $0.5 \mathrm{ml}$ of the substrate solution (isocarboxazid, $3 \mathrm{mM}$ ) was added. The incubation was continued for a further $30 \mathrm{~min}$ at $37^{\circ} \mathrm{C}$, and then enzyme activity was assayed. The concentrations of EPN. NAD and SKF 525-A used were $1 \times 10^{-5} \mathrm{M}, 5 \times 10^{-5} \mathrm{M}$ and $1 \mathrm{mM}$, respectively. Carboxylesterase activity is shown as nmoles BZH formed $/ \mathrm{mg}$ protein $/ 30 \mathrm{~min}$. Each value is the mean $\pm S$.E. from three experiments. ${ }^{*} \mathrm{P}<0.01$, statistically significant difference from the EPN-added group.

an inhibitor of dehydrogenase $(11,12)$ and cytochrome P-450 (13), this result sugested that the decrease of the $\mathrm{NAD}(\mathrm{H})$-effect by disulfiram seemed to be due to an inhibition of cytochrome P-450.

Involvement of cytochrome P-450coupled monooxygenase in NAD-effect: As shown in Table 3, NAD-effect was significantly inhibited by addition of $1 \mathrm{mM}$ of SKF 525-A (14) to the incubation mixture containing microsomes, EPN and NAD at the first $10-\mathrm{min}$ preincubation without addition of the substrate. In contrast, no inhibition of NAD-effect was seen when SKF 525-A was added at the second incubation for $30 \mathrm{~min}$ in the presence of the substrate. From these results, it was demonstrated that cytochrome P-450-coupled monooxygenase was responsible for NAD-mediated potentiation of the anti-CEase action of EPN. To confirm this, effects of KSCN on both the NAD-effect and cytochrome P-450 contents were investigated.

As shown in Fig. 4, NAD-mediated potentiation of the anti-CEase action of EPN was gradually decreased from a concentration of $0.05 \mathrm{M} \mathrm{KSCN}$ and reached to $95 \%$ inhibition at a concentration of $0.25 \mathrm{M}$, and an inverse proportional sigmoidal curve of cytochrome P-450 contents was seen under the same condition.

Effects of cetylalcohol on microsomal carboxylesterase activity: To confirm an involvement of microsomal dehydrogenase(s) in the NAD-effect, further studies were conducted.

As shown in Fig. 5, addition of cetylalcohol $\left(2.5 \times 10^{-5} \mathrm{M}\right)$ as a substrate of microsomai fatty alcohol: $\mathrm{NAD}^{+}$oxidoreductase to the incubation mixture containing microsomes, EPN and NAD $\left(1 \times 10^{-5} \mathrm{M}\right)$ caused more potent inhibition of CEase than that without added cetylalcohol. There was no significant effect of cetylalcohol when NADH instead of NAD was added. From these findings, we concluded that NAD was converted to its reduced form(NADH) through microsomal dehydrogenase(s) as a cofactor.

\section{Discussion}

Our previous studies demonstrated that the NAD-effect was observed only when liver microsomes was used as an enzyme source, and no NAD-effect was seen when pure esterase was used instead of the microsomes (2). Therefore, it is possible to speculate that some endogenous components, probably enzymes, other than CEase present in liver microsomes would be expected to be involved in the NAD-effect. To clarify the characteristics of the microsome-bound enzymes involved, liver microsomes were solubilized by using cholate at three concentrations, and the resulting pellet 


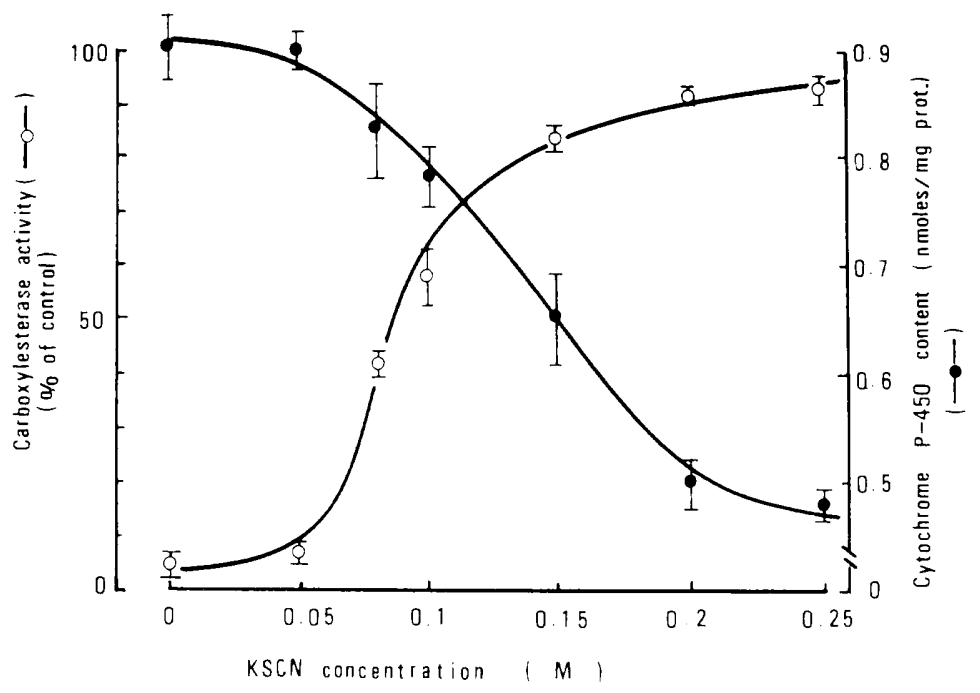

Fig. 4. Effects of KSCN on the NAD-effect and cytochrome P-450 contents. The concentrations of EPN and NAD used were $1 \times 10^{-5} \mathrm{M}$ and $5 \times 10^{-5} \mathrm{M}$, respectively. Each point is the mean $\pm S$.E. from three experiments.

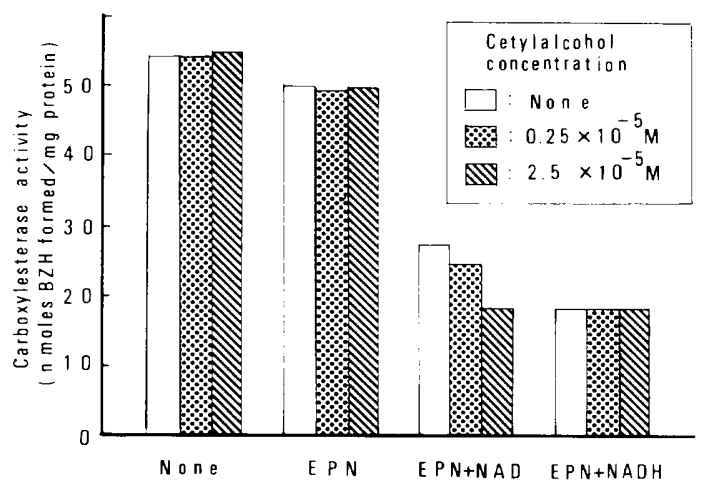

Fig. 5. Effects of cetylalcohol on the NAD-effect. The concentrations of EPN. NAD and NADH used were $1 \times 10^{-5} \mathrm{M}, 1 \times 10^{-5} \mathrm{M}$ and $5 \times 10^{-6} \mathrm{M}$, respectively. Each column is the mean of two experiments.

and the supernatant fractions were subjected to the experiments on the NAD-effect. As shown in Table 1, the NAD-effect was decreased in the pellet fraction and, conversely, increased in the supernatant fraction by addition of increasing amounts of cholate. This indicates that not only CEase but also the microsome-bound enzymes which were required for the NAD-effect were concurrently solubilized by cholate treatment under these conditions. Furthermore, the microsomal enzymes involved in the NADeffect were speculated to be dehydrogenases, because these enzymes can generate NADH from NAD, an electron donor of cytochrome P-450-coupled oxidative desulfuration of EPN to EPNoxon (14-18). To confirm this, NEM was added to the incubation mixture containing liver microsomes and EPN to block sulfhydryl groups in the dehydrogenase molecule. As expected. EPNoxon formation was completely abolished (Table 2), and CEase inhibition recovered slowly toward the control level (Fig. 2). In addition, no NADeffect was observed when disulfiram, an inhibitor of aldehyde dehydrogenase (11. 12). was added to the incubation mixture. These results demonstrated that one of the microsomal enzymes investigated could be characterized as a dehydrogenase(s) possessing sulfhydryl groups.

On the other hand, addition of SKF 525-A to the preincubation mixture in the absence of the substrate of CEase showed no NADeffect (Table 3). When KSCN was added to the incubation mixture at various concentrations, cytochrome P-450 content was gradually decreased, and conversely, potentiation of the anti-CEase action of EPN by NAD returned toward the control level (without added NAD) (Fig. 4). This demon- 
strated that NAD was enzymatically reduced to NADH by microsomal dehydrogenase(s). and subsequently, EPN was oxidized by the cytochrome P-450-coupled system with $\mathrm{NADH}$. Unlike other xenobiotics, cofactor activities of $\mathrm{NADH}$ and NADPH are almost to same extent in oxidative desulfuration of organophosphorus insecticides by cytochrome P-450-coupled monooxygenase (19-21).

Recently, Nakayasu et al. (6) reported the existence of microsomal aldehyde dehydrogenase. Further, Lee (22) demonstrated that cetylalcohol is a substrate of microsomal fatty alcohol:NAD ${ }^{+}$oxidoreductase. In this study, addition of cetylalcohol to the incubation mixture containing microsomes, EPN and NAD caused more potent inhibition of CEase than that without added alcohol. This indicates that occurrence of the NADeffect requires the conversion of NAD to $\mathrm{NADH}$ by microsomal dehydrogenase(s) prior to the enzymatic oxidation of EPN by cytochrome P-450-coupled monooxygenase. Thus, the NAD-effect is dependent upon enzymatic conversion of NAD to NADH.

The present study confirmed previous findings (2) that the amount of ${ }^{14} \mathrm{C}$-EPNoxon bound to CEase was increased by addition of NAD. That is, the NAD-effect was mainly ascribed to the increased amount of EPNoxon formed. EPN is activated by liver microsomal cytochrome P-450 into EPNoxon that covalently binds to microsomal CEase. Indeed, incubation of ${ }^{14} \mathrm{C}$-EPN, liver microsomes and NAD, but no NADPHgenerating system, resulted in the covalent binding of ${ }^{14} \mathrm{C}$ to CEase.

Figure 6 depicted a speculative diagram on the mechanism of the NAD-effect. This may be explained by four successive steps: (i) $\mathrm{NADH}$ is generated from NAD by

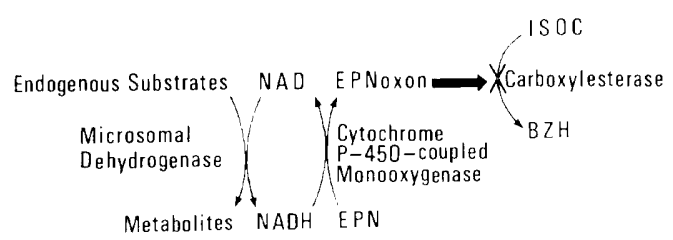

Fig. 6. Speculative diagram on the potentiation of EPN-induced inhibition of carboxylesterase by NAD. ISOC, Isocaboxazid: BZH, Benzylhydrazine. microsomal dehydrogenase(s), and then (ii) $\mathrm{NADH}$ formed is used as an electron donor of cytochrome P-450-coupled oxidative desulfuration of EPN to EPNoxon. (iii) Binding of EPNoxon formed to CEase was increased, compared to that without added NAD. Finally, (iv) CEase inhibition by EPN was increased. From the viewpoint of toxicology, CEase may be responsible for detoxication of organophosphorus insecticides. Namely, binding of oxygen analogs of these compounds to CEase, a nonvital enzyme may. reduce the anti-cholinergic action of organophosphorus insecticides.

in conclusion, taken iogether with our previous papers $(1,2)$ and unpublished observation (S. Sugiyama et al.), the present results strongly suggest that an enzymatic conversion of NAD to NADH by microsomal dehydrogenase(s) may be, at least in part, involved in binding of the organophosphate insecticides formed in vivo to CEase, by which, in turn, toxicities of these insecticides are reduced.

Acknowledgment: We are gratefully acknowledge the excellenr technical assistance of Miss Mari Takamiya.

\section{References}

1 Satoh, T., Sugiyama, S. and Kitagawa, H.: Potentiation of ethy para-nitrophenyl phenylphosphonothioate (EPN)-induced inhibition of liver microsomal carboxylesterase by NAD in vitro in rats. Res. Commun. Chem. Pathol. Pharmacol. 26, 547-562 (1979)

2 Sugiyama, S., Hosokawa, M., Igarashi, T., Ueno, K., Satoh, T. and Kitagawa, H.: Enhancement of the binding of O-ethyl O-p-nitropheny! phenylphosphonate (EPNoxon) to microsomal carboxylesterase by NAD in vitro. Japan. J. Pharmacol. 37, 39-44 (1985)

3 Imai, Y. and Sato, R.: A gel-electrophoretically homogeneous preparation of cytochrome P-450 from liver microsomes of phenobarbitalpretreated rabbits. Biocham. Biophys. Res. Commun. 60, 8-14 (1974)

4 Satoh, T. and Moroi, K.: A simple and sensitive colorimetric assay of benzylhydrazine. Anal. Chim. Acta 51, 449-454 (1970)

5 Omura, T. and Sato, R.: The carbon monoxide binding pigment of liver microsomes. J. Biol. Chem. 239, 2370-2378 (1964)

6 Nakayasu, H., Mihara, K. and Sato, R.: Purifi- 
cation and properties of a membrane-bound aldehyde dehydrogenase from rat liver microsomes. Biochem. Biophys. Res. Commun. 83, 697-704 (1978)

7 Lowry, H., Rosebrough, N.J., Farr, A.L. and Randall, R.J.: Protein measurement with the Folin phenol reagent. J. Biol. Chem. 193, 265275 (1951)

8 Sultatos, L.G. and Murphy, S.D.: Hepatic microsomal detoxification of the organophosphates paraoxon and chlorphrifos oxon in the mouse. Drug Metab. Dispos. 11, 232-238 (1983)

9 Shishido, T., Usui, K. and Fukumi, J.: Oxidative metabolism of diazinon by microsome from rat liver and cockroach fat body. Pesticide Biochem. Physiol. 2, 27-28 (1972)

10 Aldridge, W.M.: Serum esterase. 1. Two types of esterase ( $A$ and $B$ ) hydrolysing p-nitrophenyl acetate, propionate and butyrate, and a method for their determination. Biochem. J. 53, 110-117 (1953)

11 Kiedgaard, N.O.: Inhibition of aldehyde oxidase from liver by tetraethylthiuram disulphide (Antabuse). Acta Pharmacol. 5, 397-403 (1949)

12 Graham, W.D.: In vitro inhibition of liver aldehyde dehydrogenase by tetraethylthiuram disulphide. J. Pharm. Pharmacol. 3, 160-168 (1951)

13 Stripp, B., Greene, F.E. and Gillette, J.R.: Disulfiram impairment of drug metabolism by rat liver microsomes. J. Pharmacol. Exp. Ther. 170, 347-353 (1969)

14 Lasker, J.M., Graham, D.G. and Abou-Donia,
M.B.: Differential metabolism of O-ethyl O-4nitrophenyl phenylphosphonothioate by rat and chicken hepatic microsomes. Biochem. Pharmacol. 31, 1961-1967 (1982)

15 Neal, R.A. and DuBois, K.P.: Studies of the mechanism of detoxification of cholinergic phosphorothioates. J. Pharmacol. Exp. Ther. 148, 185-192 (1965)

16 Nomeir, A.A. and Dauterman, W.C.: In vitro metabolism of EPN and EPNO by mouse liver. Pesticide Biochem. Physiol. 10, 190-196 (1979)

17 Abou-Donia, M.N.: Organophosphorus esterinduced delayed neurotoxicity. Annu. Rev. Pharmacol. Toxicol. 21, 511-548 (1981)

18 Abou-Donia, M.B., Sivarajah, K. and AbouDonia, S.A.: Disposition, elimination and metabolism of O-ethyl O-4-nitrophenyl phenylphosphonothioate after subchronic application in male cats. Toxicology 26, 93-111 (1983)

19 O'Brien, R.D.: Activation of phosphorothionates by liver microsomes. Nature 183, 121-122 (1959)

20 Casida, J.E.: Specificity of substituted phenyl phosphorus compounds for esterase inhibition in mice. Biochem. Pharmacol. 5, 332-342 (1961)

21 Agosin, M., Scaramelli, N., Gil, L. and Letelier, M.E.: Some properties of the microsomal system metabolizing DDT in Triatoma infestans. Comp. Biochem. Physiol. 29, 785-793 (1969)

22 Lee, T.C.: Characterization of fatty alcohol: $\mathrm{NAD}^{+}$oxidoreductase from rat liver. J. Biol. Chem. 254, 2892-2896 (1979) 